\title{
Análisis de las pérdidas de carga en flujo turbulento en un laboratorio universitario de mecánica de fluidos
}

\author{
Dario A. Serrano*, Fainer Y. Cerpa y Gail A. Gutiérrez \\ Facultad de Ingenierías, Ingeniería Mecánica, Grupo DESTACAR, Universidad de La Guajira, Colombia \\ (correo-e: dserrano@uniguajira.edu.co; fcerpa@uniguajira.edu.co; galbeirog@uniguajira.edu.co)
}

* Autor a quien debe ser enviada la correspondencia

Recibido Oct. 8, 2020; Aceptado Dic. 7, 2020; Versión final Mar. 8, 2021, Publicado Ago. 2021

\begin{abstract}
Resumen
El objetivo del presente estudio fue desarrollar una estrategia para desarrollar un tópico clave de la mecánica de fluidos universitaria usando un enfoque teórico-experimental-computacional. Este tópico abarcó conceptos de la ecuación ampliada de Bernoulli, específicamente la obtención de las pérdidas de carga en un sistema de tuberías bajo condiciones de flujo turbulento. El aporte de la estrategia computacional resultó apropiada debido a la reducción observable en los tediosos cálculos y la optimización de los tiempos de permanencia en el laboratorio y dedicación a las prácticas. El resultado más relevante es la verificación en un ambiente universitario de las tendencias predichas por la teoría y los resultados experimentales, cuando se correlacionan en un diagrama modificado de Moody: números de Reynolds, rugosidad relativa y factor de fricción de Darcy. Se concluye que la serie de pruebas realizadas permitieron exponer las ventajas y desventajas de llevar a cabo observaciones reales y mediciones controladas en un equipo experimental junto con el análisis teórico subyacente.
\end{abstract}

Palabras clave: pérdidas de carga; rozamiento viscoso; números adimensionales; flujo turbulento; tuberías lisas; rugosa

\section{Analysis of turbulent tube flow head losses in a university fluid mechanics laboratory}

\begin{abstract}
The primary objective of this research study was to develop a strategy to examine a key topic of fluid mechanics for undergraduate education by using a theoretical-experimental-computational approach. The key topic examined covered topics from Bernoulli's extended equation, specifically, for calculating head losses in pipelines under turbulent flow conditions. The contribution of the computational approach was appropriate due to the observable reduction in both tedious calculations and optimization time for educational laboratory purposes. The most relevant result was the verification, in a university environment, of trends predicted by theory and experimental results. They correlated in a modified Moody diagram: the Reynolds number, relative roughness, and the Darcy friction factor. In conclusion, the test series showed the advantages and disadvantages of conducting real observations and controlled measurements while performing theoretical analyses.
\end{abstract}




\section{INTRODUCCIÓN}

La mecánica de fluidos estudia el comportamiento de los fluidos a través de tres leyes físicas denominadas principios de conservación de masa, momentum (leyes de Newton de la mecánica clásica) y energía (ecuación de Bernoulli) (Cengel y Cimbala, 2018), convirtiéndose en un componente curricular dentro del bloque de asignaturas de ciencias básicas aplicadas en los planes de estudios de programas en ingeniería mecánica, ingeniería civil, ingeniería ambiental, entre otros (Huilier, 2019). Su estudio es complejo porque incluye enfocarse simultáneamente en el dominio de conceptos basados en modelos matemáticos y sus comprobaciones a través de observaciones reales y mediciones controladas de variables físicas en equipos experimentales (Baldock y Chanson, 2006; Anaya-Durand et al., 2014; Garrard et al., 2020).

Hoy día, las certificaciones de calidad de los programas de ingenierías exigen la documentación de ciertos conocimientos y habilidades especificas relacionados directamente con las asignaturas mencionadas, en este caso particular es posible promover en los estudiantes el diseño de experimentos, el uso de las matemáticas aplicadas, el análisis y la interpretación de datos, el trabajo en equipo y la resolución de problemas complejos (Chowdhury, 2018; Márquez y Cárdenas, 2008). Aunque, la enseñanza de esta ciencia física puede generar interesantes debates, la literatura ha reportado diferentes puntos de vistas sobre el asunto, por ejemplo, Martin et al., (2003) propusieron el inventario de conceptos en mecánica de fluidos como una herramienta que permite descubrir las falencias en los conceptos previos que portan los estudiantes.

El comportamiento dinámico de los fluidos incluye unos tópicos relacionados con el análisis escalar de la energía mecánica e irreversibilidades en el flujo estable, incompresible e isotérmico a través de tuberías basados en los conceptos incluidos en la ecuación ampliada de Bernoulli. Algunos trabajos reportados han asumido el reto de diseñar y construir equipos experimentales para llevar a cabo comprobaciones dentro de los alcances de esta importante ecuación, siendo el agua como fluido de trabajo, el más utilizado (Alesmaeel et al., 2019; Ascencios y Poma, 2014; Macedo et al., 2001).

También cabe la posibilidad de adquirir equipos experimentales con fines didácticos a fabricantes reconocidos como Armfield y Gunt Hamburg. Sin embargo, es poco común encontrar la información suficientemente abierta y completa que permita juzgar objetivamente las discrepancias entre los análisis teóricos y los resultados experimentales correspondientes. Las causas son variadas: inestabilidad en las variables, debido a cambios amplios en el régimen de flujo y temperatura; variedad de configuraciones en los equipos experimentales; diferentes instrumentos para las mediciones de caudales y caídas de presión e inhomogeneidades en el tratamiento de los datos (Furuichi et al., 2015; Taler, 2016; Sorgun y Muftuoglu, 2020). En este trabajo se expone el análisis de una serie de pruebas realizadas en un equipo experimental estándar para estudiar los fenómenos relacionados con las pérdidas de carga en tuberías los cuales explican en parte la necesidad de los términos que ampliaron la ecuación básica de Bernoulli.

\section{OTROS ANTECEDENTES}

Las adiciones de términos a la ecuación básica de Bernoulli eliminan varias de sus restricciones para el análisis de los flujos de fluidos, transformándose en la ecuación general de la energía mecánica, la cual posibilita resolver diversos problemas prácticos (Mott y Untener, 2015):

$$
e_{1}+h_{3}-h_{4}-h_{5}=e_{2}
$$

Reemplazando las formas de la energía mecánica del fluido en las secciones extremas del sistema:

$$
\frac{P_{1}}{V}+z_{1}+\frac{v_{m 1}^{2}}{2 g}+h_{3}-h_{4}-h_{5}=\frac{P_{2}}{Y}+z_{2}+\frac{v_{m 2}^{2}}{2 g}
$$

donde, e es la energía total por unidad de peso del fluido o simplemente carga total, $\mathrm{P}$ es la presión relativa, $z$ la altura vertical a la que se encuentra el fluido, $v_{m}$ la velocidad media del fluido $(Q / A)$; $Q$ es el caudal del fluido y $A$ el área de la tubería, g la aceleración de la gravedad y y peso específico del fluido. Los términos adicionales $h_{3}, h_{4}$ y $h_{5}$ expresan las ganancias de energías por bombas y compresores, las extracciones de energía por motores y turbinas, y las pérdidas de carga debidas a las irreversibilidades por el flujo, respectivamente. Además, la Fig. 1 muestra un diagrama de un sistema característico de flujo el cual transporta un fluido desde una sección de entrada (1) hasta una sección de salida (2). 
El propósito de este estudio es en el análisis de las pérdidas de carga $h_{5}$, la cual representa las pérdidas totales (pérdidas mayores junto con pérdidas menores) en el sistema de flujo. Sin embargo, nuestro análisis se enfocará en las pérdidas mayores que se denotarán con el mismo símbolo, las cuales resultan del rozamiento viscoso (fricción) del fluido a través de su paso por el sistema de tuberías.

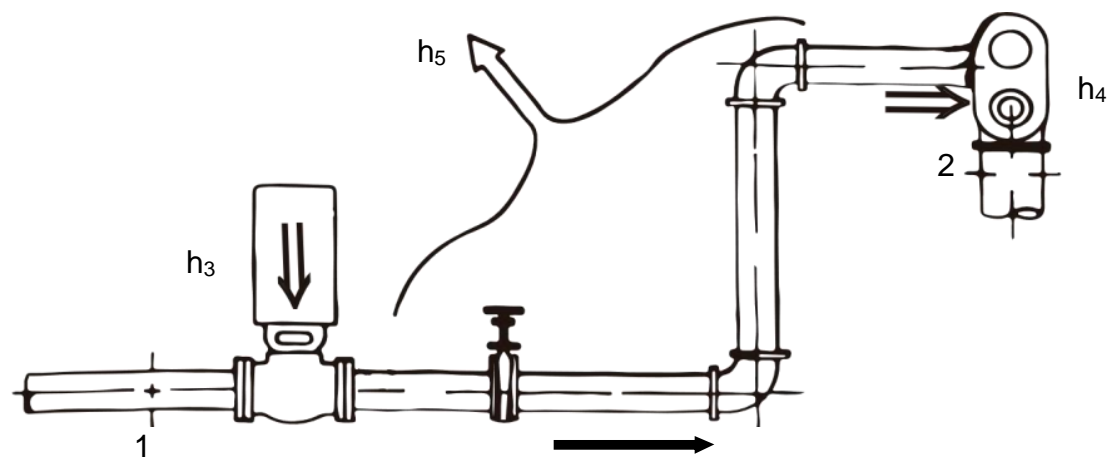

Fig. 1: Diagrama del flujo de fluido para la aplicación de ecuación general de la energía. Adaptada de Mott y Untener, 2015

\section{Pérdidas de carga por fricción en tuberías}

El análisis de las pérdidas mayores universalmente utiliza el modelo matemático desarrollado por los ingenieros Henry Darcy y Julius Weisbach en la década de los años veinte. La ecuación dimensionalmente homogénea denominada DW se expresa en términos de la pérdida de carga en la tubería, así (Cengel y Cimbala, 2018; Crane, 1992; Lahiouel y Lahiouel, 2015):

$$
h_{5}=f_{D} \cdot \frac{L}{D} \cdot \frac{v_{m}^{2}}{2 g}
$$

También, se puede representar en términos de una caída de presión (ec. 4). Donde, fo es el factor de fricción de Darcy, L la longitud de la sección de prueba, $D$ el diámetro interno de la tubería y $\rho$ la densidad del fluido.

$$
\Delta P=f_{D} \cdot \frac{L}{D} \cdot \frac{\rho \cdot v_{m}^{2}}{2}
$$

En la practica la dificultad de esta ecuación DW se centra en la determinación del factor de fricción (Cengel y Cimbala, 2018). Sin embargo, en la literatura es posible encontrar diferentes modelos matemáticos tanto de carácter implícitos como explícitos en función de dos números adimensionales: número de Reynolds $(\operatorname{Re})$ y la rugosidad relativa de la tubería (k/D), para obtener el factor de fricción (Offor y Alabi, 2016; Zeghadnia et al., 2019; Minhoni et al., 2020). Una clasificación estándar del régimen de flujo de fluidos en tuberías circulares es (Cengel y Cimbala, 2018): $R e<2300$ (Régimen laminar); $2300<R e<4000$ (Régimen de transición); y Re>4000 (Régimen turbulento). El número de Reynolds se define de la siguiente forma (ec. 5), donde, $\mu$ es la viscosidad dinámica del fluido.

$$
\operatorname{Re}=\frac{\rho \cdot v_{m} \cdot D}{\mu}
$$

\section{Ecuación universal de Colebrook-White}

El rango de aplicabilidad se encuentra en el intervalo de $4 \times 10^{3} \leq R e \leq 10^{8}, 0 \leq k / D \leq 5 \times 10^{-2}$. Su universalidad es debida a que predice muy bien los casos límites de tuberías lisas con $\mathrm{k} / \mathrm{D} \rightarrow 0 \mathrm{y}$ tuberías completamente rugosas con $\mathrm{Re} \rightarrow \infty$. Tal nivel de predicción se logra en los casos prácticos que consideran tuberías comerciales (Colebrook y White, 1937; Colebrook, 1939). La forma de la ecuación CW es:

$$
\frac{1}{\sqrt{f_{D}}}=-2.0 \cdot \ln \left(\frac{\frac{k}{D}}{3.71}+\frac{2.51}{\operatorname{Re} \sqrt{f_{D}}}\right)
$$


Su carácter implícito es un inconveniente para resolver en términos del factor de fricción desconocido; sin embargo, hoy día se tiene acceso fácil a métodos numéricos robustos y rápidos. En esta investigación se usó un método de bisección en un muy útil formato vectorial, el cual inicia la búsqueda en un intervalo fijo definido en $(0,008)$ para el factor de fricción (Sartorius, 2015). Debido a tal complejidad de la ecuación CW, muchos autores han realizado correlaciones para proponer ecuaciones con menos dificultad de cálculo, sin tener que usar métodos numéricos, siendo así de carácter explícito en función del número de Reynolds (Brkić, 2011; Fang et al., 2011; Fang et al., 2020). A pesar de esto, la ecuación CW ha sido las más precisas hasta el momento para el cálculo del factor de fricción, tanto para tuberías lisas como rugosas.

\section{METODOLOGÍA}

El trabajo tiene un enfoque cuantitativo con un estudio correlacional basado en los análisis teórico, experimental y numérico. Los ensayos con dos réplicas en las diferentes líneas de conducción del equipo (lisas y rugosa), fueron considerados para su análisis aplicando métodos numéricos computacionales y así comparar los resultados experimentales contra los teóricos.

\section{Descripción del equipo}

El equipo para el estudio detallado de pérdidas de carga en tuberías, según el fabricante, contiene los siguientes elementos (ver Fig. 2), tanque de almacenamiento (1), escala de niveles (2), bomba centrífuga (3), válvula de globo (4) permite regular el flujo al sistema, válvulas de bolas (5), tuberías lisas de diferentes diámetros (6), tubería con rugosidad artificial (7), puntos para tomas de presiones (8) y termómetro (9). El estudio de cada tubería de manera separada implicada la apertura total de la válvula de bola correspondientes. Además, cada tubería tiene una sección de prueba; como se observa en la Fig. 3 , permitiendo el análisis de las pérdidas de carga en la sección media con una longitud fija de 1.0 metro por simplicidad (la relación mínima L/D fue de 58.6 y la máxima de 225.2). El diseño del equipo considero la restricción para flujo totalmente desarrollado en las entradas a las secciones de pruebas, lo cual implico considerar longitudes superiores a 10 diámetros antes de los puntos de inicio de medición. El equipo experimental funciona en un régimen de caudal máximo hasta $80 \mathrm{lpm}$, presión máxima hasta $1500 \mathrm{mmHg}$ y un rango de Reynolds entre $10^{3}$ y $10^{5}$.



Fig. 2: Esquema del equipo utilizado que permite el estudio detallado de las pérdidas de carga en tuberías. 


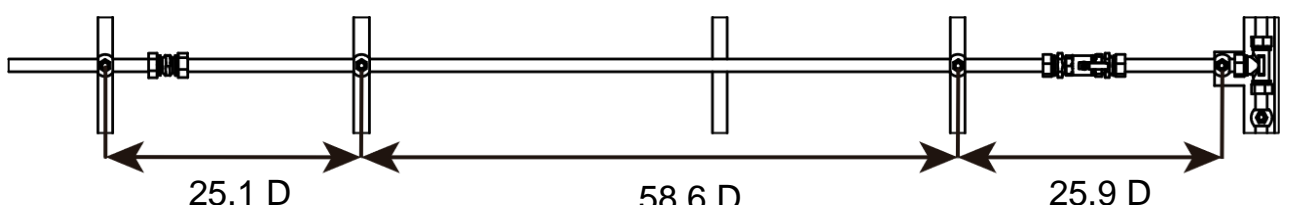

Fig. 3: Esquema de la sección de prueba para este estudio.

\section{Medición de las variables de flujo}

La medición de flujo volumétrico o caudal $(\dot{Q})$ fue a través de la determinación del volumen en un tanque calibrado a ciertos intervalos de tiempo. El tanque cuenta con dos escalas de volúmenes, una de 0 a 6.0 litros (intervalos de 1.0) y otra 0 a 40.0 litros (intervalos de 5.0). Cada una de las escalas permite indicación instantánea mediante el nivel de agua dentro del tanque; usándolo en conjunto con un cronómetro fue posible determinar el volumen instantáneo en la escala junto con el intervalo de tiempo respectivo. Dos réplicas, permitieron promediar los tiempos para cada volumen instantáneo, haciendo posible ajustar linealmente por mínimos cuadrados la curva de volumen vs tiempo; la pendiente de la línea ajustada arrojó el caudal. Se controló que los coeficientes de correlación del ajuste lineal en cada medición de flujo se mantuvieran por encima de 0.98. Otros requerimientos tenidos en cuenta para lograr estas mediciones fueron controlar que la temperatura del agua no aumentara exageradamente, la toma de datos lo más rápidas posibles, entre otros. Este método de medición del caudal hace que el proceso general de toma de datos se ralentice en exceso (ISO4185, 1993).

La medición de la caída de presión en la sección de prueba fue leída en una escala con lectura digital, lo que permitió promedios integrados y lecturas de picos: máximo y mínimo. La escala de medición usada fue 0 $1500 \mathrm{mmHg}$ y con una resolución de $0.01 \mathrm{mmHg}$. Las mediciones de la temperatura, se tomaron al inicio y fin de una prueba, adicionalmente se usó con el propósito de estimar las propiedades de densidad y viscosidad del agua en estado de líquido saturado mediante ayuda de software (Holmgren, 2006).

\section{Implementación de software a la medida}

En el diseño del software se tuvieron en cuenta los siguientes procesos, la lectura de la variables de entrada implico el diámetro interno de la tubería $(\mathrm{mm})$, longitud de sección de prueba $(\mathrm{cm})$, temperatura inicial y final $\left({ }^{\circ} \mathrm{C}\right)$, parejas de volúmenes y tiempos de llenado $(\mathrm{l}, \mathrm{s})$, caída de presión mínima y máxima $(\mathrm{mmHg})$, y para las variables de salida implicaron el caudal (lpm), número de Reynolds (adimensional), presión media (mmHg), velocidad media $(\mathrm{m} / \mathrm{s})$, factores de fricción teórico y experimental (adimensional) y error experimental (\%).

Internamente el software llevo a cabo los cálculos de las propiedades del agua como la densidad y la viscosidad con la presión atmosféricas y la temperatura media del agua mediante la función Xstream (Holmgren, 2006). El caudal fue el resultado del ajuste lineal de las parejas de volúmenes y tiempos usando la función fit. El número de Reynolds mediante la Ec. 5. El factor de fricción teórico despajándolo de la Ec. (6) mediante el método de bisección en formato vectorizado (Sartorius, 2015). El factor de fricción experimental despejándolo de la Ec. 4. La especificación de la rugosidad relativa para el caso de las tuberías lisas se asumió nula, mientras que para el caso de la tubería rugosa hubo la necesidad de tantear su valor de los resultados experimentales. Para el despliegue de graficas el software utiliza 6 mediciones por tuberías de diferentes caudales. Todos estos procedimientos de cálculos y de graficas fue integrado en un software utilizando una interfaz de usuario simple e intuitiva tal como se muestra en la Fig. 4. El software puede permitir la reducción de cálculos repetitivos disminuyendo los tiempos de uso del laboratorio y la dedicación al desarrollo de las prácticas.

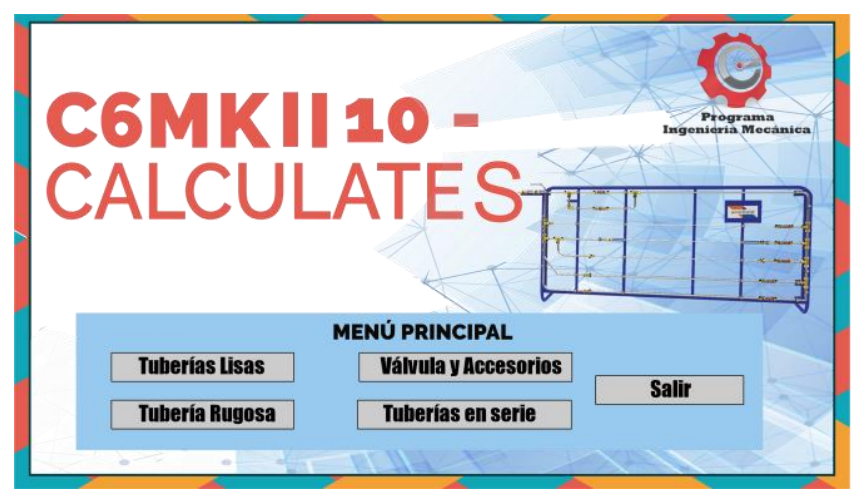

Fig. 4: Ventana principal del software a la medida 


\section{RESULTADOS}

El análisis detallado de las pérdidas de cargas tuvo como objetivo principal el estudio de tuberías lisas con diferentes diámetros y una con rugosidad artificial, para un total de 5 tuberías. Cada tubería funcionó en un rango de operación que se expone en la Tabla 1. Las escalas usadas para la medición de caudales fueron: escala inferior: $1.0-8.0 \mathrm{lpm}$ y la escala superior: $8.0-55 \mathrm{lpm}$. Las tuberías lisas presentan comportamientos cualitativos similares como se hará evidente en las Figs. 5 y 6.

Tabla 1: Rango operacional de cada tubería.

\begin{tabular}{|c|c|c|c|}
\hline Tubería & $\begin{array}{c}\Delta \mathrm{P} \\
(\mathrm{mmHg})\end{array}$ & $\begin{array}{c}\dot{\mathrm{Q}} \\
(\mathrm{Ipm})\end{array}$ & $\begin{array}{c}\text { Re } \\
\text { (Adimensional) }\end{array}$ \\
\hline $\begin{array}{c}\text { Lisa No. } 1 \\
\mathrm{D}=4.44 \mathrm{~mm}\end{array}$ & $33.4-698.5$ & $1.028-6.243$ & $5.9 \times 10^{3}-3.5 \times 10^{4}$ \\
\hline $\begin{array}{c}\text { Lisa No. } 2 \\
\mathrm{D}=7.64 \mathrm{~mm}\end{array}$ & $15.1-765.2$ & $3.003-26.982$ & $1.0 \times 10^{4}-9.7 \times 10^{4}$ \\
\hline $\begin{array}{c}\text { Lisa No. } 3 \\
\mathrm{D}=10.96 \mathrm{~mm}\end{array}$ & $13.1-306.9$ & $5.808-38.710$ & $1.4 \times 10^{4}-9.4 \times 10^{4}$ \\
\hline $\begin{array}{c}\text { Lisa No. } 4 \\
\mathrm{D}=17.07 \mathrm{~mm}\end{array}$ & $4.3-55.7$ & $11.659-52.250$ & $1.9 \times 10^{4}-8.6 \times 10^{4}$ \\
\hline $\begin{array}{c}\text { Rugosa No. } 5 \\
\mathrm{D}=15.17 \mathrm{~mm}\end{array}$ & $10.0-211.2$ & $9.230-42.740$ & $1.6 \times 10^{4}-7.4 \times 10^{4}$ \\
\hline
\end{tabular}

En la Fig. 5 se puede observar la comparación del número de Reynolds contra la caída de presión, donde los datos experimentales se representan en marcadores (\$); para la tubería 4 y los resultados teóricos a través de la Ec. 6 con rugosidad relativa $k / D=0$, se representan con la línea continua (-) de perdida de carga teórica para la misma tubería.

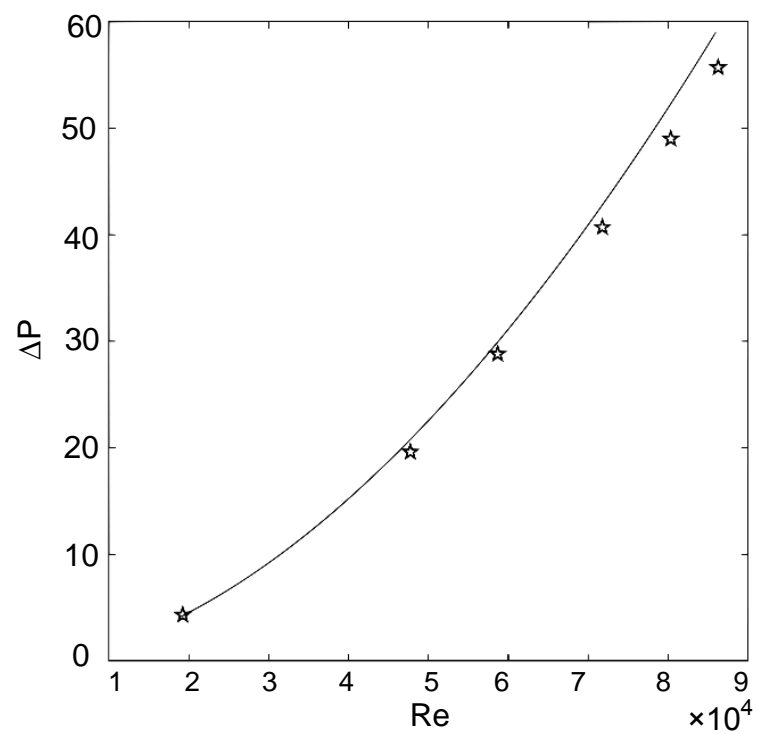

Fig. 5: Caída de presión vs número de Reynolds en tubería No. 4.

En la Fig. 6 se puede observar la comparación entre el número de Reynolds contra el factor de fricción (fD), donde los resultados experimentales se encuentran a través de la Ec. 4 y se representan en marcadores ( y los resultados teóricos mediante la Ec. 6 con rugosidad relativa $\mathrm{k} / \mathrm{D}=0$, se representan mediante una línea continua (-). Además, nuestros resultados fueron comparados con datos de alta precisión expuestos por Furuichi et al., 2015, representados en marcadores (o). Se puede observar que existe una tendencia aceptable entre nuestros resultados. 


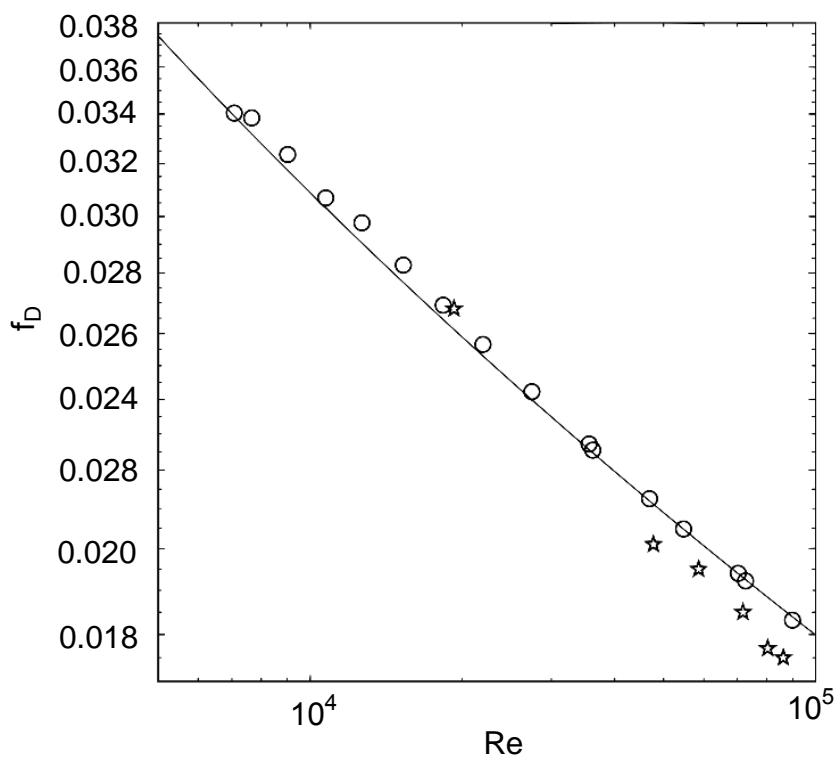

Fig. 6: Factores de fricción vs número de Reynolds en tubería No. 4.

Finalmente, en la Fig. 7 mediante la relación funcional entre $f_{D}^{-1 / 2}$ vs $\operatorname{Re} \cdot f_{D}^{1 / 2}$ en escala semilog a partir de un diagrama de Moody modificado, los resultados experimentales se representaron como: tubería 1 (x), tubería $2\left(^{*}\right)$, tubería $3(\diamond)$, tubería $4(\star \star *)$, tubería $5(\triangleleft)$ y los resultados teóricos como: ecuación CW con k/D=0 (-), ecuación $\mathrm{CW}$ con $\mathrm{k} / \mathrm{D}=0.027$ (- -), ecuación $\mathrm{CW}$ con $\mathrm{k} / \mathrm{D}=0.019$ (-.) y ecuación $\mathrm{CW}$ con k/D=0.023 (.). También, se tuvieron en cuenta los datos tomados de Furuichi et al., 2015 (o). Se reconoce que los datos de las tuberías No. 1 al 4 tienen un comportamiento de tubería lisa (aproximadamente sin rugosidad) en régimen turbulento. Por otra parte, la tubería con rugosidad artificial se aleja de tal comportamiento con valores de rugosidad relativa alrededor de $2 \%$. Similarmente, se expone los datos de la referencia (Furuichi et al., 2015), estos predicen con mayor exactitud el modelo teórico usado en un amplio rango de regímenes de flujo. Según nuestros resultados experimentales se estima que el nivel promedio de incertidumbre respecto al modelo teórico fue de $8.2 \%$ para el régimen turbulento en tubería lisa. Mientras que los datos de referencia (Furuichi et al., 2015) los cuales reclaman alta precisión, estiman el nivel de incertidumbre en un $2.5 \%$.

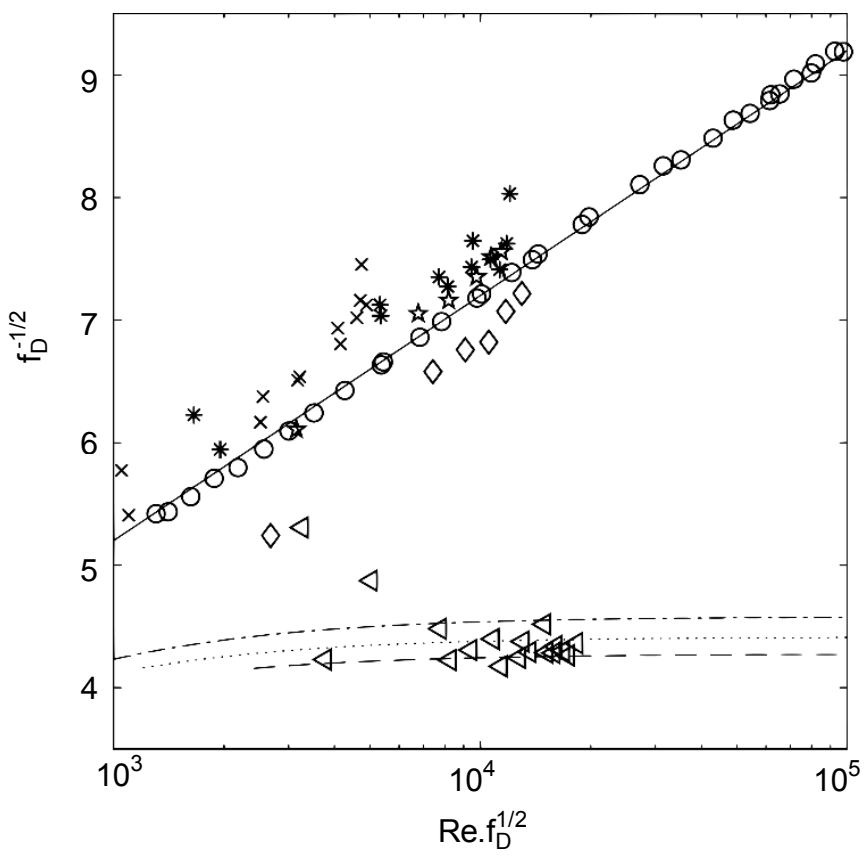

Fig. 7: Diagrama modificado de Moody en escala semilog. 


\section{DISCUSIÓN}

El presente estudio teórico-experimental logró evaluar las pérdidas de carga por fricción en tuberías tanto lisa como rugosa, lo cual tiene gran importancia conceptual en cualquier curso básico de mecánica de fluidos. Los resultados obtenidos experimentalmente cuando se comparan con el modelo teórico muestran un comportamiento cualitativamente similar, tal que el comportamiento global de los datos de las tuberías lisas coincide con lo esperado. Así mismo se muestran los datos del comportamiento de la tubería rugosa estudiada. Las fuentes de los errores pueden enfocarse en las escasas sensibilidad y calibración que tienen los medidores de flujo y presión. Sin embargo, los resultados bajo el diseño experimental propuesto forman un conjunto de datos de referencia para posibles mejoras y aplicación en casos similares que implican el transporte de fluidos dentro de tuberías.

Hoy en día, existen códigos muy accesibles y eficientes que facilitan y aceleran los análisis cubiertos en este trabajo: herramientas de visualización, buscadores de raíces y bases de datos de propiedades de los fluidos con un uso sencillo. Adicionalmente, permite desmitificar a estos niveles del pregrado la compresión, construcción y aplicación del diagrama de Moody.La comparación realizada con datos experimentales confiables traza unas posibles mejoras para llevar a cabo en los laboratorios universitarios. También, un código computacional con su interfaz de usuario resultó como producto de este trabajo para apoyar los análisis en el laboratorio, el cual se registró ante la Dirección Nacional de Derechos de Autor - DNDA, con el número 13-64-379 y fecha 05-dic-2017.

\section{CONCLUSIONES}

La serie de pruebas realizadas permitieron exponer las ventajas y desventajas de llevar a cabo observaciones reales y mediciones controladas en un equipo experimental junto con el análisis teórico subyacente. Resultó especialmente satisfactorio verificar las tendencias predichas por la teoría y los resultados experimentales, cuando se correlacionan en un diagrama modificado de Moody el número de Reynolds, la rugosidad relativa y el factor de fricción de Darcy. Igualmente resaltar la utilidad de los números adimensionales para simplificar el tratamiento que involucra un número amplio de variables físicas. Cálculos relativamente simples pero monótonos son requeridos para obtener las pérdidas de carga en un sistema de tuberías bajo diversas condiciones de flujos, las cuales habilitan el uso de la ecuación ampliada de Bernoulli, central en la dinámica de fluidos universitaria. Una estrategia que resultó relevante fue incluir el desarrollo de software a la medida, siendo posible automatizar los tediosos cálculos y optimizar los tiempos de permanencia en el laboratorio y dedicación a las prácticas. Adicionalmente, este producto fue protegido ante las autoridades competente de derechos de autor. El trabajo pendiente es la inserción de esta estrategia en los cursos de mecánica de fluidos de nuestra universidad. La cual resultará conveniente debido a que posibilita levantar registros de una serie de evidencias concretas para las certificaciones de calidad educativa que se están pretendiendo lograr.

\section{AGRADECIMIENTOS}

La realización de este trabajo contó con el invaluable apoyo en temas como logística y financiero por parte de Sistema Integral de Laboratorios (SILAB) y la Dirección de Investigaciones de la Universidad de La Guajira.

\section{REFERENCIAS}

Alesmaeel, M., Alfarsi, A., y otros seis autores, Analysis of a Hydraulic Pipe System with Major and Minor Pressure Losses, https://doi.org/10.17265/1934-7359/2019.03.006, Journal of Civil Engineering and Architecture, 13 (3), $209-217$ (2019).

Anaya-Durand, A.I., Cauich-Segovia, G.I., y otros dos autores, Evaluación de Ecuaciones de Factor de Fricción Explícito para Tuberías, https://doi.org/10.1016/S0187-893X(14)70535-X, Educación Química, 25 (2), 128-134 (2014).

Ascencios, D., y Poma, R., Diseño y construcción de un banco de ensayo para el estudio de pérdidas de carga por fricción y singularidad, http://dx.doi.org/10.21704/ac.v75i1.941, Anales Científicos, 75 (1), 116-124 (2014).

Baldock, T.E., y Chanson, H., Undergraduate Teaching of Ideal and Real Fluid Flows: The Value of Real-World Experimental Projects, https://doi.org/10.1080/03043790600911837, European Journal of Engineering Education, 31 (6), 729-739 (2006).

Brkić, D., New Explicit Correlations for Turbulent Flow Friction Factor, https://doi.org/10.1016/j.nucengdes.2011.07.042, Nuclear Engineering and Design, 241 (9), 4055-4059 (2011).

Cengel, Y., y Cimbala, J., Mecánica de Fluidos: Fundamentos y Aplicaciones, 4ª Ed., 1-1025, McGraw-Hill, Ciudad de México, México (2018).

Chowdhury, H., Alam, F. and Mustary, I., Development of an innovative technique for teaching and learning of laboratory experiments for engineering courses, https://doi.org/10.1016/j.egypro.2019.02.154, Energy Procedia, 160, 806 - 111 (2019). 
Colebrook, C.F., y White, C.M., Experiments with Fluid Friction in Roughened Pipes, https://doi.org/10.1098/rspa.1937.0150, Proceedings of the Royal Society A: Mathematical, Physical and Engineering Sciences, 161 (906), 367-381 (1937).

Colebrook, C.F., Turbulent Flow in Pipes, with Particular Reference to the Transition Region between the Smooth and Rough Pipe Laws, https://doi.org/10.1680/ijoti.1939.13150, J Inst Civ. Eng, 11 (4), 133-156 (1939).

Crane, Flujo de Fluidos: en Válvulas: Accesorios y Tuberías, 1ª Ed., 1-215, McGraw-Hill, Ciudad de México, México (1992).

Fang, X., Xu, Y. and Zhou, Z., New Correlations of Single-Phase Friction Factor for Turbulent Pipe Flow and Evaluation of Existing Single-Phase Friction Factor Correlations, https://doi.org/10.1016/j.nucengdes.2010.12.019, Nucl. Eng. Des., 241 (3), 897-902 (2011).

Fang, X., Xu, L., y otros dos autores, Correlations for Friction Factor of Turbulent Pipe Flow under Supercritical Pressure: Review and a New Correlation, https://doi.org/10.1016/j.pnucene.2019.103085, Progress in Nuclear Energy, 118, 103085 (2020)

Furuichi, N., Terao, Y., y otros dos autores, Friction Factor and Mean Velocity Profile for Pipe Flow at High Reynolds Numbers, https://doi.org/10.1063/1.4930987, Physics of Fluids, 27,1-15 (2015).

Garrard, A., Bangert, K. and Beck, S., Large-Scale, Multidisciplinary Laboratory Teaching of Fluid Mechanics, Fluids, https://doi.org/10.3390/fluids5040206, 5 (4), 206 (2020).

Holmgren, M., X Steam for Matlab, www.X-eng.com, 1-5 (2006).

Huilier, D., Forty Years' Experience in Teaching Fluid Mechanics at Strasbourg University, https://doi.org/10.3390/fluids4040199, Fluids, 4 (4), 199 (2019).

ISO 4185:1980, Technical Corrigendum, Measurement of Liquid Flow in Closed Conduits - Weighing Method, 1-21, Suiza (1993).

Lahiouel, Y., y Lahiouel, R., Evaluation of Energy Losses in Pipes, https://doi.org/10.12691/ajme-3-3A-6, American Journal of Mechanical Engineering, 3 (3A), 32-37 (2015).

Macedo, R.C., Martínez, J.J., y Vélez, J.F., Diseño, Construcción y Validación de una Unidad Piloto para el Manejo de Fluidos Newtonianos, Información Tecnológica, 12 (6), 169-176 (2001).

Márquez, D., y Cárdenas, O., Implementación de un Laboratorio Virtual para la enseñanza de Controladores PID, http://dx.doi.org/10.4067/S0718-07642008000300011, Información tecnológica, 19 (3), 75-78 (2008).

Martin, J., Mitchell, J. and Newell, T., Development of a concept inventory for fluid mechanics, https://doi.org/10.1109/FIE.2003.1263340, 33rd Annual Frontiers in Education, 1, T3D-23 - T3D-28 (2003).

Minhoni, R., Pereira, F., y otros tres autores, The performance of explicit formulas for determining the Darcy-Weisbach friction factor, http://dx.doi.org/10.1590/1809-4430-eng.agric.v40n2p258-265/2020, Eng. Agríc., 40 (2), $258-265$ (2020).

Mott, R., y Untener, J., Mecánica de Fluidos, $7^{a}$ Ed., 1-647, PEARSON, Ciudad de México, México (2015).

Offor, U. H., and Alabi, S. B., An Accurate and Computationally Efficient Explicit Friction Factor Model, https://doi.org/10.4236/aces.2016.63024, Advances in Chemical Engineering and Science, 6 (3), 237-245 (2016).

Sartorius, S., Bisection Method Root Finding, MathWorks, (2015). [Online]

Sorgun, M., y Muftuoglu, T.D., A New Friction Factor Formula for Single Phase Liquid Flow Through Geothermal Pipelines, https://doi.org/10.1016/j.geothermics.2020.101901, Geothermics, 88, 101901 (2020).

Taler, D., Determining Velocity and Friction Factor for Turbulent Flow in Smooth Tubes, https://doi.org/10.1016/j.ijthermalsci.2016.02.011, International Journal of Thermal Sciences, 105, 109-122 (2016).

Zeghadnia, L., Robert, J. L. and Achour, B., Explicit solutions for turbulent flow friction factor: A review, assessment and approaches classification, https://doi.org/10.1016/j.asej.2018.10.007, Ain Shams Engineering Journal, 10 (1), $243-252$ (2019). 
